\title{
Evaluation of IFN- $\gamma$ Enzyme-linked Immunospot Assay (ELISPOT) as a First-line Test in the Diagnosis of Non-Immediate Hypersensitivity to Amoxicillin and Penicillin
}

\author{
Lenka Sedláčková ${ }^{1}$, Miroslav Průcha ${ }^{1}$, Ingrid Poláková ${ }^{2}$ Blanka Míková1 \\ ${ }^{1}$ Department of Clinical Biochemistry, Haematology and Immunology, $\mathrm{Na}$ Homolce \\ Hospital, Prague, Czech Republic; \\ ${ }^{2}$ Department of Immunology, Institute of Haematology and Blood Transfusion, \\ Prague, Czech Republic
}

Received December 29, 2017; Accepted March 19, 2018.

Key words: ELISPOT - Allergy - Hypersensitivity - Penicillin - Amoxicillin

Abstract: The current diagnostic algorithm for beta-lactam allergy is based on skin and provocation tests, both of which carry a certain risk of inducing hypersensitivity reactions. Thus, non-invasive in vitro tests reliable enough to replace skin and provocation tests at least in a portion of patients are desirable. We aimed to verify the utility of IFN- $\gamma$ ELISPOT as a first-line test in patients with suspected non-immediate hypersensitivity reaction to amoxicillin (AMX) and penicillin (PNC). The prospective observational study included 24 patients with recent, suspected non-immediate hypersensitivity reaction to AMX or PNC and 6 recently-exposed healthy subjects. In vitro tests were performed in all patients and healthy subjects: a) IFN- $\gamma$ ELISPOT with PNC, AMX and amoxicillin plus clavulanic acid (AMX-CL); b) penicillin specific $\mathrm{IgE}$; c) basophil activation test (BAT). Skin and provocation tests followed only in certain patients. IFN- $\gamma$ ELISPOT results with PNC and AMX stimulation did not differ from the unstimulated condition. The highest IFN- $\gamma$ responses to $\mathrm{AMX}-\mathrm{CL}$ were close to previously published criteria in three patients;

This study was supported by the Ministry of Health of the Czech Republic - conceptual development of the research organisation (Nemocnice Na Homolce - NNH, Prague, Czech Republic, 00023884), IG144102.

Mailing Address: Lenka Sedláčková, MD., Department of Clinical Biochemistry, Haematology and Immunology, Na Homolce Hospital, Roentgenova 2, 15030 Prague 5, Czech Republic; Phone: +420 257272 823; e-mail: lenka.sedlackova@homolka.cz 
one of which had true hypersensitivity according to drug provocation tests. Five patients with confirmed hypersensitivity by skin tests showed no response to the culprit antibiotic on IFN- $\gamma$ ELISPOT assay. Our results did not support the utility of IFN- $\gamma$ ELISPOT in the diagnosis of mild, non-immediate hypersensitivity to amoxicillin and penicillin.

\section{Introduction}

Beta-lactam hypersensitivity has a significant impact on treatment options in affected patients. Up to $10 \%$ of patients have a self-reported allergy to penicillin. The avoidance of penicillin and other beta-lactam antibiotics increases the consumption of second-line broad-spectrum antibiotics and is followed by many unfavourable consequences: higher rate of treatment failures and complications, increased microbial resistance and higher treatment costs (Macy and Contreras, 2014; Solensky, 2014). However, true penicillin hypersensitivity is known to be present in only $10 \%$ of patients labelled penicillin-allergic. The correct determination of penicillin allergy has become a very important issue in public health.

Beta-lactam hypersensitivity has heterogeneous manifestations. From a clinical and diagnostic point of view, the most useful classification distinguishes between immediate and non-immediate hypersensitivity. Immediate hypersensitivity generally begins within 1 hour from exposure to an antibiotic and is often mediated by immunoglobulin $\mathrm{E}(\mathrm{IgE})$. Non-immediate hypersensitivity manifests later, usually within several hours or days, and is primarily mediated by T-lymphocytes (Blanca et al., 2009). The current diagnostic algorithm for beta-lactam hypersensitivity recommended by the European Network of Drug Allergy (ENDA) and the Drug Allergy Interest Group (DAIG) of the European Academy of Allergy and Clinical Immunology (EAACl) relies mostly on in vivo tests (skin and provocation tests) (Demoly et al., 2014), while the role of in vitro tests is complementary. The availability of complex allergy diagnostics by in vivo testing is currently suboptimal in the Czech Republic (CZ) as it carries certain risks for patients, and thus is provided in only a few experienced allergy centres. Currently, a simple approach to screening suspected beta-lactam allergy in vitro is used for immediate hypersensitivity in CZ, mainly using specific lgE, as well as the basophil activation test (BAT) to lesser extent. When a positive result confirms sensitisation and corresponds to clinical history, further examination is not required. All negative results should be followed up as the sensitivity of specific lgE and BAT for betalactam hypersensitivity is low (Demoly et al., 2014; Mayorga et al., 2016). Most beta-lactam hypersensitivity reactions are non-immediate, therefore the ability to confirm at least some cases by in vitro methods is desirable.

As non-immediate hypersensitivity reactions to beta-lactams are mediated primarily by T-lymphocytes, in vitro determination is focused on the detection of beta-lactam specific T-lymphocytes. Various tests of lymphocyte proliferation, 
activation or cytokine production have been employed. Among other techniques, enzyme-linked immunospot assay (ELISPOT) appears to be very promising, as it is an exceptionally sensitive method for the detection of specific T-lymphocytes. Peripheral blood mononuclear cells (PBMC) obtained from a sensitised patient are cultured in the presence of the suspected drug. Re-stimulated lymphocytes secrete cytokines that are trapped by a membrane-captured cytokine-specific primary antibody and visualised by an enzyme-linked secondary antibody, similar to ELISA. The result is expressed as spot forming cells (= number of cytokine-secreting cells). ELISPOT is capable of detecting < 25 secreting cells per million PBMC (Ebo et al., 2011; Mayorga et al., 2016). Interferon $\gamma($ IFN- $\gamma$ ) is considered a key cytokine in the pathogenesis of delayed drug hypersensitivity reactions, especially maculopapular exanthemas (Rozieres et al., 2009; Porebski and Czarnobilska, 2015). A study by Rozieres et al. (2009) reported a sensitivity of $91 \%$ and specificity of $95 \%$ for IFN- $\gamma$ ELISPOT in patients with maculopapular exanthema after amoxicillin treatment. We aimed to confirm the utility of IFN- $\gamma$ ELISPOT as a first-line in vitro test in the diagnosis of amoxicillin non-immediate hypersensitivity, as well as in non-immediate penicillin hypersensitivity.

\section{Material and Methods}

Twenty-five patients (group A) and 6 healthy subjects (group B) were enrolled in the prospective observational study between September 2014 and June 2016. Group characteristics are presented in Tables 1, 2 (group A) and 3 (group B). The inclusion criterion for group $A$ (patients) was manifestations of a non-immediate hypersensitivity reaction with an onset of 1 hour or more from the initiation of penicillin (PNC) or amoxicillin (AMX) treatment within the last year. The inclusion criterion for group $B$ (healthy subjects) was well-tolerated PNC or AMX treatment within the last year. Both common forms of $A M X$ treatment were included: $A M X$ alone and $A M X$ plus clavulanic acid (AMX-CL). The study was approved by the Ethics Committee of $\mathrm{Na}$ Homolce Hospital, and all participants provided signed, informed consent.

In vitro tests for each participant were performed at one time. The timing was targeted to between one month and one year from the resolution of the hypersensitivity reaction in group $A$ and between one month and one year from the end of antibiotic treatment in group B. Three sets of tests were performed in all subjects: a) IFN- $\gamma$ ELISPOT to detect T-lymphocytes specific to benzylpenicillin, AMX and AMX-CL; b) penicillin-specific IgE; c) BAT. Specific IgE and BAT was included to detect the incidental presence of type IgE sensitisation to penicillin beta-lactams.

\section{ELISPOT assay}

For the detection of drug-specific IFN- $\gamma$ producing T-lymphocytes, a pre-coated Human IFN- $\gamma$ ELISPOT Kit (C.T.L. Europe GmbH, Bonn, Germany) was used. 


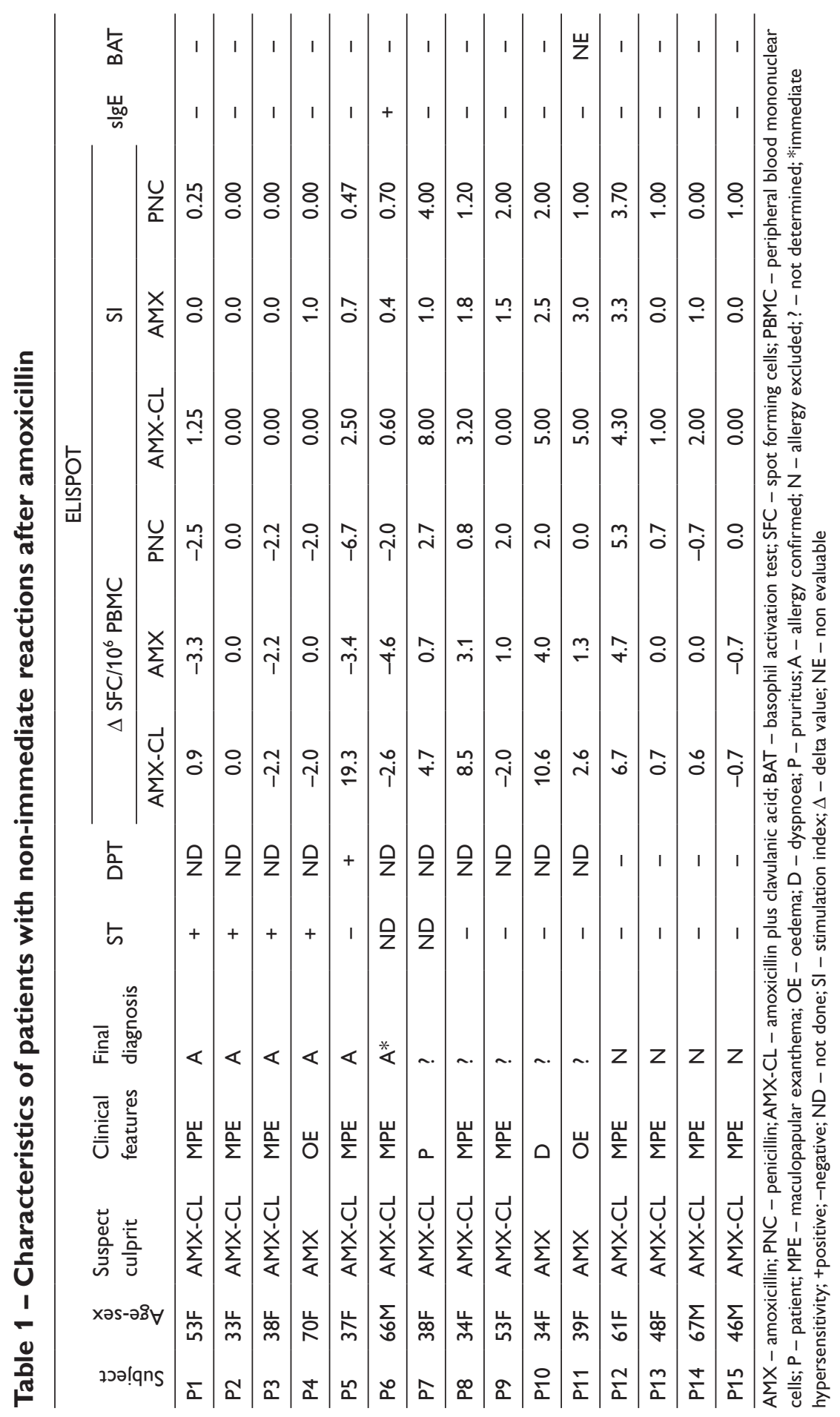




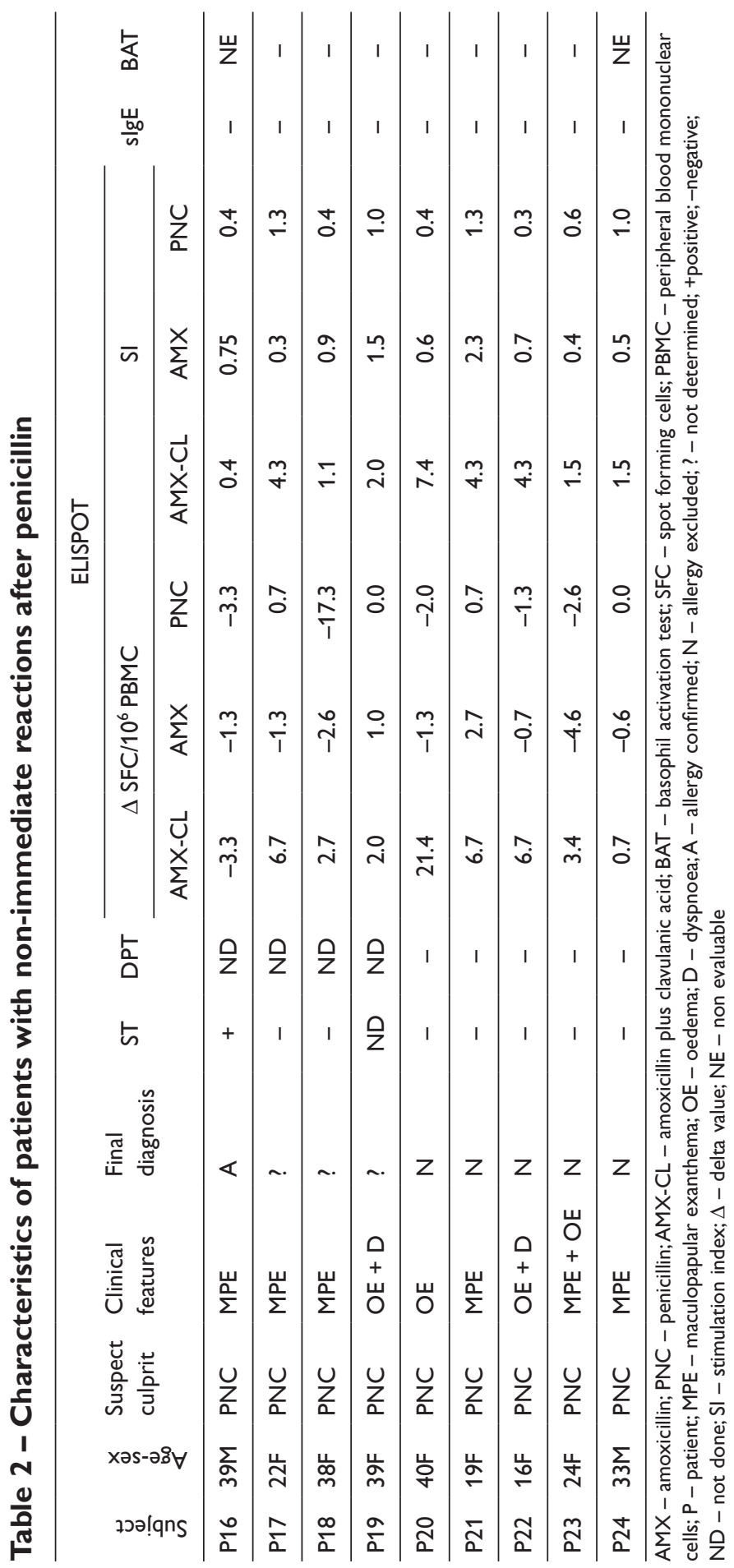


Table 3 - Characteristics of healthy controls

\begin{tabular}{|c|c|c|c|c|c|c|c|c|c|c|}
\hline \multirow{3}{*}{$\begin{array}{l}\frac{\breve{\omega}}{0} \\
\frac{\tilde{\omega}}{\vec{n}}\end{array}$} & \multirow{3}{*}{ 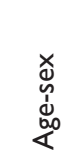 } & \multirow{3}{*}{$\begin{array}{l}\text { Tolerated } \\
\text { beta- } \\
\text { lactam }\end{array}$} & \multicolumn{6}{|c|}{ ELISPOT } & \multirow{3}{*}{ slgE } & \multirow{3}{*}{ BAT } \\
\hline & & & \multicolumn{3}{|c|}{$\Delta \mathrm{SFC} / 10^{6} \mathrm{PBMC}$} & \multicolumn{3}{|c|}{ SI } & & \\
\hline & & & $\mathrm{AMX}-\mathrm{CL}$ & AMX & PNC & $\mathrm{AMX}-\mathrm{CL}$ & AMX & PNC & & \\
\hline $\mathrm{HC} 1$ & $54 \mathrm{~F}$ & $\mathrm{AMX}-\mathrm{CL}$ & 7.8 & -2.2 & -10.0 & 0.0 & 0.8 & 0.0 & - & - \\
\hline $\mathrm{HC} 2$ & $60 \mathrm{~F}$ & $A M X-C L$ & 6.7 & -3.3 & -10.0 & 1.3 & 0.8 & 0.5 & - & - \\
\hline $\mathrm{HC} 3$ & $50 \mathrm{~F}$ & $\mathrm{AMX}-\mathrm{CL}$ & 2.0 & 0.6 & -0.7 & 4.0 & 2.0 & 0.0 & - & - \\
\hline $\mathrm{HC} 4$ & $36 \mathrm{~F}$ & $\mathrm{AMX}-\mathrm{CL}$ & 1.3 & 0.7 & 0.7 & 0.0 & 0.0 & 0.0 & - & - \\
\hline $\mathrm{HC} 5$ & $44 \mathrm{~F}$ & PNC & 0.0 & 0.0 & 0.0 & 0.0 & 0.0 & 0.0 & - & - \\
\hline $\mathrm{HC} 6$ & $21 \mathrm{~F}$ & PNC & -1.4 & -1.4 & 0.0 & 0.5 & 0.5 & 1.0 & - & - \\
\hline
\end{tabular}

AMX - amoxicillin; PNC - penicillin; AMX-CL - amoxicillin plus clavulanic acid; BAT - basophil activation test; SFC - spot forming cells; PBMC - peripheral blood mononuclear cells; $\mathrm{HC}$ - healthy control; $\mathrm{SI}$ - stimulation index; $\Delta$ - delta value; +positive; -negative

Blood was drawn into a Cell Preparation Tube (Vacutainer ${ }^{\circledR}$ CPT $^{\text {TM }}$ Heparin, Becton Dickinson). Peripheral blood mononuclear cells (PBMC) were isolated after centrifugation at $1500 \mathrm{~g}$ and their concentration was adjusted to $5 \times 10^{6}$ cells $/ \mathrm{ml}$ by adding serum-free CTL Test ${ }^{\mathrm{TM}}$ Medium (C.T.L. Europe GmbH, Bonn, Germany). The assay was performed in triplicate. PBMC $\left(100 \mu \mathrm{l}, 5 \times 10^{5} /\right.$ well $)$ were incubated with benzylpenicillin (Penicilin $\mathrm{G}$ draselna sol ${ }^{\circledR}$ Biotika, $100 \mu$ l, concentration $5 \times 10^{5} \mathrm{IU} / \mathrm{ml}$ ), AMX-CL (Amoksiklav ${ }^{\circledR}$ Lek Pharmaceuticals, $100 \mu \mathrm{l}$, concentration $1 \mathrm{mg} / \mathrm{ml}$ ) and AMX (Amoxicillin for skin tests, Diater, $100 \mu \mathrm{l}$, concentration $1 \mathrm{mg} / \mathrm{ml}$ ) for 24 hours at $37{ }^{\circ} \mathrm{C}$ in $5 \% \mathrm{CO}_{2}$. The antibiotic concentration used was adapted from Rozieres et al. (2009). As a negative control, cells were incubated with medium (100 $\mu \mathrm{l} \mathrm{CTL} \mathrm{medium),} \mathrm{and} \mathrm{phytohemagglutinin} \mathrm{(PHA,} \mathrm{Sigma} \mathrm{Aldrich,}$ St Louis, USA, $100 \mu \mathrm{l}$, concentration $5 \mu \mathrm{g} / \mathrm{ml}$ ) stimulation was used as a positive control. The cells were then removed by two washes with PBS (phosphate buffered saline) and two washes with PBS $-0.05 \%$ Tween-20, IFN- $\gamma$ detection antibody was added for 2 hours, the wells were washed three times with PBS $-0.05 \%$ Tween- 20 . Streptavidin-AP Solution was added for $30 \mathrm{~min}$, followed by two washing steps with PBS $-0.05 \%$ Tween- 20 and with distilled water. Spots were developed using the Developer Solution and the reaction was stopped by washing three times with tap water. Spot forming cells (SFC) were counted by a CTL-ImmunoSpot S5 UV Analyzer (C.T.L. Europe GmbH, Bonn, Germany). Results were expressed as: a) the number of IFN- $\gamma$ SFC $/ 10^{6}$ PBMC for the unstimulated condition (medium) and stimulation with each antibiotic (AMX-CL, AMX and PNC); b) delta $(\Delta)$ values, the difference between the response value in the presence of antibiotic and the unstimulated condition value (Tables 1-3); c) stimulation index (SI), response value in the presence of the antibiotic divided by the unstimulated value in each participant (Tables 1-3). 
Specific lgE to penicilloyl G, penicilloyl V, ampicilloyl and amoxicilloyl was performed by ImmunoCAP assay (ThermoFisher Scientific, Uppsala, Sweden).

$B A T$ was performed by the FlowCAST assay (Bühlmann, Basel, Switzerland), with benzylpenicilloyl-L-octa-lysin, sodium benzylpenilloate and sodium amoxicillin (Diater, Madrid, Spain), penicillin G (Penicilin G draselna sol ${ }^{\circledR}$, Biotika), ampicillin (Ampicilin ${ }^{\circledR}$, Biotika) and amoxicillin plus clavulanic acid (Amoksiklav ${ }^{\circledR}$, Lek Pharmaceuticals). BAT was analysed by a flow cytometer FACS Calibur (BD Biosciences, San Jose, USA).

\section{Follow-up}

Clinical evaluation by skin and provocation tests followed in one subgroup of patients. Skin tests (ST) with major and minor penicillin determinant (benzylpenicilloyl-L-octa-lysin and sodium benzylpenilloate, DAP Diater, Madrid, Spain), penicillin G (Penicilin G draselna sol ${ }^{\circledR}$, Biotika, $10000 \mathrm{IU} / \mathrm{ml}$ ) and amoxicillin + clavulanic acid (Amoksiklav ${ }^{\circledR}$ Lek Pharmaceuticals, $20 \mathrm{mg} / \mathrm{ml}$ ) were performed in 21 patients according to the ENDA and DAIG of EAACI recommendations (Brockow et al., 2013). A drug provocation test (DPT) with culprit beta-lactam was performed in 10 patients; in 5 cases with oral phenoxymethylpenicillinum (V-Penicilin ${ }^{\circledR}$, Biotika) and in 5 cases with amoxicillin + clavulanic acid (Amoksiklav ${ }^{\circledR}$, Lek Pharmaceuticals). The DPTs were performed as described previously (Romano et al., 2004).

\section{Statistical analysis}

Normality was evaluated using a chi-square test for each variable. As normal data distribution was not shown by the chi-square test, the Wilcoxon paired test was used to evaluate the significance testing between patient group results for unstimulated conditions and stimulation with each antibiotic. P-values $<0.05$ were considered statistically significant.

\section{Results}

IFN- $\gamma$ ELISPOT assay results were evaluated in 24 of 25 patients from group A and in all 6 healthy subjects from group B. The number of IFN- $\gamma$ producing PBMC showed strong response to PHA (positive control) in all but one patient, who was therefore excluded from further analyses. Antibiotic response was generally weak, very close to the unstimulated condition (incubation with medium alone). Results expressed as delta $(\Delta)$ values (number of IFN- $\gamma$ SFC/10 $10^{6}$ PBMC cultured with each antibiotic after subtraction of values obtained from PBMC cultured with medium only) are presented in Table 1 for patients reacting to amoxicillin, Table 2 for patients reacting to penicillin and Table 3 for healthy controls tolerating amoxicillin or penicillin. Often the number of IFN- $\gamma$ SFC was slightly higher in wells with medium than with the antibiotic, and therefore some delta values were less than zero. The stimulation indices (SI) were also calculated (Tables 1-3) and data 
Table 4 - Characteristics of results

\begin{tabular}{lcccc}
\hline $\begin{array}{l}\text { Group } A \\
(\mathrm{n}=24)\end{array}$ & $\begin{array}{c}\text { Medium } \\
\left(\mathrm{SFC} / 10^{6} \mathrm{PBMC}\right)\end{array}$ & $\begin{array}{c}\mathrm{AMX}-\mathrm{CL} \\
\left(\mathrm{SFC} / 10^{6} \mathrm{PBMC}\right)\end{array}$ & $\begin{array}{c}\text { AMX } \\
\left(\mathrm{SFC} / 10^{6} \mathrm{PBMC}\right)\end{array}$ & $\begin{array}{c}\mathrm{PNC} \\
\left(\mathrm{SFC} / 10^{6} \mathrm{PBMC}\right)\end{array}$ \\
\hline Min-max & $0-31.3$ & $0-34$ & $0-28.7$ & $0-14$ \\
Median & 2 & 4.45 & 2 & 2 \\
Interquartile range & $1-3.6$ & $1-9.7$ & $0.7-4.35$ & $0.7-4.7$ \\
Chi-square test & reject normality & reject normality & reject normality & reject normality
\end{tabular}

AMX - amoxicillin; PNC - penicillin; AMX-CL - amoxicillin plus clavulanic acid; SFC - spot forming cells;

PBMC - peripheral blood mononuclear cells

Table 5 - Statistical significance by Wilcoxon test

\begin{tabular}{ll}
\hline Group A $(n=24)$ & P-value \\
\hline AMX-CL vs. medium & $0.0071^{*}$ \\
AMX vs. medium & 0.5217 \\
PNC vs. medium & 0.2253 \\
\hline
\end{tabular}

AMX - amoxicillin; PNC - penicillin; AMX-CL - amoxicillin plus clavulanic acid; * $\mathrm{p}<0.05$

characteristics are summarised in Table 4. Group comparison (drug stimulation versus unstimulated condition) is presented in Table 5. A significant difference in IFN- $\gamma$ secretion was found only between PBMC cultured with medium and PBMC cultured with AMX-CL. Results obtained with AMX and benzylpenicillin did not differ significantly from the unstimulated condition (medium).

IgE sensitization was detected in one patient (patient No. 6) by specific IgE positivity to penicilloyl V (7.58 kUA/l), ampicilloyl (1.46 kUA/l), penicilloyl G $(0.74 \mathrm{kUA} / \mathrm{l})$ and amoxicilloyl $(0.71 \mathrm{kUA} / \mathrm{l})$. None of the patients or healthy subjects had positive BAT; the results of 3 patients were not evaluated, in one case due to high spontaneous activation and in two cases due to the absence of basophils in the analysis (Tables 1 and 2).

Follow-up by skin and provocation tests confirmed drug hypersensitivity in 6 patients and excluded it in 9 patients (Tables 1 and 2). Nine patients did not finish the allergy work up. Positive DPT corresponded to the second highest IFN- $\gamma$ response to AMX-CL (patient No. 5). Five patients had hypersensitivity confirmed by ST, but showed no response to the culprit antibiotic in the IFN- $\gamma$ ELISPOT assay (patients No. 1, 2, 3, 4 and 16).

\section{Discussion}

The utility of ELISPOT in the diagnosis of drug hypersensitivity has been shown by several authors (Rozieres et al., 2009; Zawodniak et al., 2010; El-Ghaiesh et al., 2012; Esser et al., 2012; Fu et al., 2012; Polak et al., 2013; Tanvarasethee et al., 2013; 
Ben-Said et al., 2015; Kato et al., 2017; Trubiano et al., 2017), as well as in many case reports. Nevertheless, the target patient population, drug used, cytokine studied and protocols differ considerably. Also, the reported sensitivity for beta-lactams varies over a wide range, from 13 to $91 \%$.

The objective of the present study was to verify the efficacy of ELISPOT in non-immediate beta-lactam hypersensitivity. It was previously reported that the IFN- $\gamma$ ELISPOT assay shows good sensitivity (91\%) and specificity (95\%) in demonstrating delayed drug hypersensitivity reactions to amoxicillin (Rozieres et al., 2009). We aimed to confirm its utility as a first-line test not only for amoxicillin, but also for penicillin. Skin and provocation tests are routinely used at our department, however patient compliance to the entire procedure is generally suboptimal as it is time-consuming and not risk free. As the sensitivity of in vivo and in vitro drug allergy tests decreases over time (Fernández et al., 2009), an inclusion criterion of reaction or exposure to penicillin or amoxicillin in the last 12 months was used.

We used very similar conditions to those described previously (Rozieres et al., 2009). However, our study did not confirm the previous findings. None of our patients achieved the suggested cut-off value for positivity, $30 \mathrm{SFC} / 10^{6}$ PBMC (Rozieres et al., 2009). Assays with PNC (generic Penicilin G draselna sol $^{\circledR}$ Biotika) and sole AMX (commercial Amoxicillin for skin tests, Diater) in the same concentration as in the original work failed completely; the difference between unstimulated conditions and drug stimulation was negligible. The assay with AMX-CL (generic Amoksiklav ${ }^{\circledR}$ Lek Pharmaceuticals) significantly differed from unstimulated conditions and some results stood out among others. Selective hypersensitivity to clavulanic acid is not a probable explanation as the culprit antibiotic did not contain clavulanic acid in two of three patients with the highest IFN- $\gamma$ response to AMX-CL. Selective hypersensitivity to clavulanic acid is also not as common in $\mathrm{CZ}$ as was recently reported in immediate hypersensitivity patients by Torres et al. (2016) in Spain. More likely, some drugs are stronger stimulators than others (Khalil et al., 2008) and differences may exist between epitope availability in their particular forms. Further studies elucidating this issue are needed.

Some authors have suggested increasing the sensitivity of ELISPOT by detecting a wider range of cytokines, e.g. IFN- $\gamma$ plus IL-4, IL-5, IL-13 and granzyme B (Beeler et al., 2006; Zawodniak et al., 2010; El-Ghaiesh et al., 2012; Polak et al., 2013; Tanvarasethee et al., 2013; Mayorga et al., 2016). Protocol modifications regarding the length of incubation, pre-treatment of PBMC with cytokines, monoclonal antibodies or dendritic cells and other amplification strategies have been proposed. A novel study by Kato et al. (2017) revealed improvement of IFN- $\gamma$ ELISPOT sensitivity when PBMCs were stimulated by CD3/CD28 and IL-2 for 7 days. They found 17 out of 20 samples positive by the modified ELISPOT, while only 4 out of 20 were positive by the conventional ELISPOT. 
ELISPOT is more sensitive to severe forms of hypersensitivity, e.g. in drug rash with eosinophilia and systemic symptoms (DRESS), Stevens-Johnson syndrome (SJS) or toxic epidermal necrolysis (TEN), in comparison to maculopapular exanthema (Fu et al., 2012; Ben-Said et al., 2015). A recent study by Trubiano et al. (2017) found $53 \%$ sensitivity for conventional IFN- $\gamma$ ELISPOT in antibiotic-associated severe cutaneous adverse reactions (DRESS, SJS, TEN and acute generalized exanthematous pustulosis), with a median $102 \mathrm{SFC} / 10^{6} \mathrm{PBMC}$ and interquartile range (IQR) 71.46-147.3 in positive cases. Skin test sensitivity was $52 \%$ and the combination of ST and ELISPOT allowed the identification of antibiotic causality in 79\%. The benefit of ELISPOT was apparent, especially in acute samples from DRESS patients and in glycopeptide-associated cases (vancomycin and teicoplanin). Our patients did not experience severe manifestations. Our findings are in accordance with those of Khalil et al. (2008), who reported only weak response (between 3 to 5 spots) in patients with maculopapular exanthema following amoxicillin using IFN- $\gamma$ ELISPOT.

Fifteen of 24 patients completed the full examination necessary for final diagnosis. We confirmed non-immediate hypersensitivity to amoxicillin or amoxicillin plus clavulanic acid in 5 patients; in 4 by ST, in 1 by DPT (Table 1). We excluded nonimmediate hypersensitivity to amoxicillin plus clavulanic acid in 4 patients by ST and DPT.We confirmed hypersensitivity to penicillin in 1 patient by ST and excluded it in 5 patients by ST and DPT. No serious complications were recorded during ST or DPT. Six patients had negative skin tests but refused or did not complete provocation tests. IgE type sensitisation was recorded in 1 patient by specific IgE to all four penicillins tested, its confirmation by ST or DPT was not performed. Thus, our group of patients $(A)$ consisted of 6 patients with confirmed non-immediate hypersensitivity to AMX (1 AMX alone, 4 AMX and/or $C L)$ or PNC (1), 1 patient with immediate hypersensitivity to penicillin and aminopenicillins, 9 patients with excluded hypersensitivity and 8 patients in which the final diagnosis was not established.

We did not find any significant results with IFN- $\gamma$ ELISPOT using published criteria under very similar conditions (Rozieres et al., 2009). Using softer evaluation criteria with a combination of $\mathrm{SI}(>2)$ and delta values (>10 SFC/106 PBMC, higher than our healthy subjects) would lead to 3 positive results with $A M X-C L$ and zero positive results with $A M X$ alone or $P N C$. In patient No. 5, the $A M X-C L$ result (19.3 SFC/10 ${ }^{6}$ PBMC, SI 2.5) corresponded to DPT. In patient No. 20, the AMX-CL result (21.4 SFC/106 $\mathrm{PBMC}, \mathrm{SI} 7.4)$ did not correspond to the reaction culprit (PNC; PNC result was $1.3 \mathrm{SFC} / 10^{6} \mathrm{PBMC}, \mathrm{SI} 0.6$ ) and hypersensitivity to both, PNC and AMX-CL, was eventually excluded by DPT. The third potentially significant result (patient No. 10; $\left.\mathrm{AMX}-\mathrm{CL} 10.6 \mathrm{SFC} / 10^{6} \mathrm{PBMC}, \mathrm{SI} 5\right)$ remains unvalidated, as the patient had negative ST but did not completed DPT. IFN- $\gamma$ ELISPOT did not help to omit in vivo testing in any patient. The highest result (in patient No. 20) even suggests a risk of false positivity if using more lenient criteria. 
The present study has some limitations. The study design reflected the intended use of IFN- $\gamma$ ELISPOT as a first-line test, so patient enrolment was based on clinical suspicion and not on hypersensitivity confirmation by ST and DPT. Therefore, the patient group was "diluted" by subjects without true hypersensitivity and the number of definitively confirmed cases was small. The eight patients lost to follow up reflect the common issue of poor compliance with complicated time-consuming procedures and concerns about safety. We did not create our own cut-off values for IFN- $\gamma$ ELISPOT evaluation. As normal data distribution was not shown, we were unable to use mean +2 SD (standard deviation) to establish cut-off values using assays incubated under unstimulated conditions. Moreover, due to the small number of healthy participants in the present study, it would be inappropriate to create cut-offs from antibiotic assays in these subjects.

We did not assess the exact sensitivity and specificity of the IFN- $\gamma$ ELISPOT method for each antibiotic studied due to the small number of patients with a final diagnosis. According to our findings, the sensitivity of the assay appears to be much lower than the optimistic expectations based on the work of Rozieres et al. (2009). With 1 potentially positive result in 6 confirmed hypersensitivity cases, we may assume an approximate sensitivity to be less than $20 \%$ in mild non-immediate hypersensitivity reactions to penicillin and amoxicillin using an assay with AMX-CL. The failure of PNC and AMX alone requires further research focused on the particular form of beta-lactam employed in the assay.

\section{Conclusion}

Our results did not support the utility of IFN- $\gamma$ ELISPOT in the diagnosis of mild non-immediate hypersensitivity to amoxicillin and penicillin in daily practice. The first-line diagnostic approach remains a combination of detailed history and in vivo tests. Skin tests are the most useful tests for allergy confirmation. After ST negativity or even without performing ST in mild childhood MPE (maculopapular exanthema), the gold standard of drug allergy diagnosis is a drug provocation test. DPT is considerably safe in non-severe non-immediate cases and it is the only test able to exclude hypersensitivity. Possible benefits of in vitro tests including IFN- $\gamma$ ELISPOT in more severe forms of non-immediate beta-lactam hypersensitivity (DRESS, SJS, TEN) or in patients with contraindications to ST and DPT due to comorbidities requires further research and validation. Sensitivity enhancement of ELISPOT by protocol modification may be helpful.

\section{References}

Beeler, A., Engler, O., Gerber, B. O., Pichler,W. J. (2006) Long-lasting reactivity and high frequency of drug-specific T cells after severe systemic drug hypersensitivity reactions. J. Allergy Clin. Immunol. 117(2), 455-462.

Ben-Said, B., Arnaud-Butel, S., Rozières, A., Rodet, K., Bérard, F., Nicolas, J. F., Nosbaum, A. (2015) Allergic delayed drug hypersensitivity is more frequently diagnosed in drug reaction, eosinophilia and systemic 
symptoms (DRESS) syndrome than in exanthema induced by beta-lactam antibiotics. J. Dermatol. Sci. 80(1), 71-74.

Blanca, M., Romano, A., Torres, M. J., Férnandez, J., Mayorga, C., Rodriguez, J., Demoly, P., Bousquet, P. J., Merk, H. F., Sanz, M. L., Ott, H., Atanaskovic-Markovic, M. (2009) Update on the evaluation of hypersensitivity reactions to betalactams. Allergy 64, 183-193.

Brockow, K., Garvey, L. H., Aberer, W., Atanaskovic-Markovic, M., Barbaud, A., Bilo, M. B., Bircher, A., Blanca, M., Bonadonna, B., Campi, P., Castro, E., Cernadas, J. R., Chiriac, A. M., Demoly, P., Grosber, M., Gooi, J., Lombardo, C., Mertes, P. M., Mosbech, H., Nasser, S., Pagani, M., Ring, J., Romano, A., Scherer, K., Schnyder, B., Testi, S., Torres, M., Trautmann, A., Terreehorst, I.; ENDA/EAACI Drug Allergy Interest Group (2013) Skin test concentrations for systemically administered drugs - an ENDA/EAACI Drug Allergy Interest Group position paper. Allergy 68(6), 702-712.

Demoly, P., Adkinson, N. F., Brockow, K., Castells, M., Chiriac, A. M., Greenberger, P. A., Khan, D. A., Lang, D. M., Park, H. S., Pichler, W., Sanchez-Borges, M., Shiohara, T., Thong, B. Y. (2014) International Consensus on drug allergy. Allergy 69, 420-437.

Ebo, D. G., Leysen, J., Mayorga, C., Rozieres, A., Knol, E. F., Terreehorst, I. (2011) The in vitro diagnosis of drug allergy: status and perspectives. Allergy 66, 1275-1286.

El-Ghaiesh, S., Monshi, M. M., Whitaker, P., Jenkins, R., Meng, X., Farrell, J., Elsheikh, A., Peckham, D., French, N., Pirmohamed, M., Park, B. K., Naisbitt, D. J. (2012) Characterization of the antigen specificity of T-cell clones from piperacillin-hypersensitive patients with cystic fibrosis. J. Pharmacol. Exp. Ther. 341(3), 597-610.

Esser, S., Jablonka, R., Heinemann, F. M., Reuter, S., Jaeger, H., von Krosigk, A., Schenk-Westkamp, P., Schadendorf, D., Horn, P. A., Lindemann, M. (2012) Detection of abacavir hypersensitivity by ELISpot method. Inflamm. Allergy Drug Targets 11(3), 227-234.

Fernández, T. D., Torres, M. J., Blanca-López, N., Rodríguez-Bada, J. L., Gomez, E., Canto, G., Mayorga, C., Blanca, M. (2009) Negativization rates of IgE radioimmunoassay and basophil activation test in immediate reactions to penicillins. Allergy 64(2), 242-248.

Fu, M., Gao, Y., Pan, Y., Li, W., Liao, W., Wang, G., Li, C., Li, C., Gao, T., Liu, Y. (2012) Recovered patients with Stevens-Johnson syndrome and toxic epidermal necrolysis maintain long-lived IFN- $\gamma$ and sFasL memory response. PLoS One 7(9), e45516.

Kato, K., Kawase, A., Azukizawa, H., Hanafusa, T., Nakagawa, Y., Murota, H., Sakaguchi, S., Asada, H., Katayama, I. (2017) Novel interferon- $\gamma$ enzyme-linked immunoSpot assay using activated cells for identifying hypersensitivity-inducing drug culprits. J. Dermatol. Sci. 86(3), 222-229.

Khalil, G., El-Sabban, M., Al-Ghadban, S., Azzi, S., Shamra, S., Khalifé, S., Maroun, R. (2008) Cytokine expression profile of sensitized human $\mathrm{T}$ lymphocytes following in vitro stimulation with amoxicillin. Eur. Cytokine Netw. 19(3), 131-141.

Macy, E., Contreras, R. (2014) Health care use and serious infection prevalence associated with penicillin "allergy" in hospitalized patients: a cohort study. J. Allergy Clin. Immunol. 133, 790-796.

Mayorga, C., Celik, G., Rouzaire, P., Whitaker, P., Bonadonna, P., Rodrigues-Cernadas, J., Vultaggio, A., Brockow, K., Caubet, J. C., Makowska, J., Nakonechna, A., Romano, A., Montanez, M. I., Laguna, J. J., Zanoni, G., Gueant, J. L., Oude Elberink, H., Fernandez, J., Viel, S., Demoly, P., Torres, M. J.; In vitro tests for Drug Allergy Task Force of EAACI Drug Interest Group (2016) In vitro tests for drug hypersensitivity reactions: an ENDA/EAACI Drug Allergy Interest Group position paper. Allergy 71, 1103-1134.

Polak, M. E., Belgi, G., McGuire, C., Pickard, C., Healy, E., Friedmann, P. S., Ardern-Jones, M. R. (2013) In vitro diagnostic assays are effective during the acute phase of delayed-type drug hypersensitivity reactions. Br. J. Dermatol. 168(3), 539-549. 
Porebski, G., Czarnobilska, E. (2015) Drug-specific in vitro secretion of IFN $\gamma$ in the diagnosis of drug-induced exanthemas: electrochemiluminiscence assay versus previously used diagnostic methods. Przegl. Lek. 72(12), 721-724.

Romano, A., Blanca, M., Torres, M. J., Bircher, A., Aberer, W., Brockow, K., Pichler, W. J., Demoly, P. (2004) Diagnosis of nonimmediate reactions to beta-lactam antibiotics. Allergy 59(11), 1153-1160.

Rozieres, A., Hennino, A., Rodet, K., Gutowski, M. C., Gunera-Saad, N., Berard, F., Cozon, G., Bienvenu, J., Nicolas, J. F. (2009) Detection and quantification of drug-specific T cells in penicillin allergy. Allergy 64(4), 534-542.

Solensky, R. (2014) Penicillin allergy as a public health measure. J. Allergy Clin. Immunol. 133, 797-798.

Tanvarasethee, B., Buranapraditkun, S., Klaewsongkram, J. (2013) The potential of using enzyme-linked immunospot to diagnose cephalosporin-induced maculopapular exanthems. Acta Derm. Venereol. 93(1), 66-69.

Torres, M. J., Montanez, M. I., Ariza, A., Salas, M., Fernandez, T. D., Berbero, N., Mayorga, C., Blanca, M. (2016) The role of IgE recognition in allergic reactions to amoxicillin and clavulanic acid. Clin. Exp. Allergy 46, 264-274.

Trubiano, J. A., Strautins, K., Redwood, A. J., Pavlos, R., Konvinse, K. C., Aung, A. K., Slavin, M. A., Thursky, K. A., Grayson, M. L., Phillips, E. J. (2017) The combined utility of ex vivo IFN- $\gamma$ release enzyme-linked immunoSpot assay and in vivo skin testing in patients with antibiotic-associated severe cutaneous adverse reactions. J. Allergy Clin. Immunol. Pract. (Epub ahead of print)

Zawodniak, A., Lochmatter, P., Yerly, D., Kawabata, T., Lerch, M., Yawalkar, N., Pichler, W. J. (2010) In vitro detection of cytotoxic $T$ and NK cells in peripheral blood of patients with various drug-induced skin diseases. Allergy 65(3), 376-384. 